\title{
Proper co-ordinates of non-inertial observers and rotation
}

\author{
Hrvoje Nikolić \\ Theoretical Physics Division, Rudjer Bošković Institute, \\ P.O.B. 180, HR-10002 Zagreb, Croatia \\ hrvoje@thphys.irb.hr
}

December 4, 2018

\begin{abstract}
By proper co-ordinates of non-inertial observers (shortly - proper non-inertial coordinates) we understand the proper co-ordinates of an arbitrarily moving local observer. After a brief review of the theory of proper non-inertial co-ordinates, we apply these co-ordinates to discuss the relativistic effects seen by observers at different positions on a rotating ring. Although there is no relative motion among observers at different positions, they belong to different proper non-inertial frames. The relativistic length seen by an observer depends only on his instantaneous velocity, not on his acceleration or rotation. For any observer the velocity of light is isotropic and equal to $c$, provided that it is measured by propagating a light beam in a small neighbourhood of the observer.
\end{abstract}

\section{Proper non-inertial co-ordinates}

In physics, all dynamical equations of motion are certain differential equations that describe certain quantities as functions of space-time points. Space-time points are parametrized by their co-ordinates. It is convenient to write the equations of motion (as well as other related equations) in a form which is manifestly covariant with respect to general co-ordinate transformations. When one solves the equations, one must use some specific co-ordinates. The covariance provides that one can use any co-ordinates he wants, because later he can easily transform the results to any other co-ordinates. Therefore, it is convenient to choose co-ordinates that simplify the technicalities of the physical problem considered.

The general covariance is often interpreted as a statement that "physics does not depend on the co-ordinates chosen". However, this is not so. The choice of co-ordinates is more than a matter of convenience. The main purpose of theoretical physics is to predict what will be observed under given circumstances. The main lesson we have learned from Lorentz 
co-ordinates is the fact that what an observer observes (time intervals, space intervals, components of a tensor, ...) depends on how the observer moves. Lorentz co-ordinates are proper co-ordinates of an observer that moves inertially in flat space-time. Proper non-inertial coordinates are the generalization of Lorentz co-ordinates to arbitrary motion in arbitrary space-time. If one is interested in how a physical system looks like to a specific observer, one must transform the results to the corresponding proper non-inertial co-ordinates.

Proper non-inertial co-ordinates are determined by the (time-like) trajectory of the observer, by the rotation of the observer with respect to a local inertial observer and by the properties of space-time itself. The general geometrical construction of proper non-inertial co-ordinates is well established [1]. Here I present the most important properties of proper non-inertial co-ordinates:

1. Proper non-inertial co-ordinates are chosen such that the position of the observer is given by $x^{\mu}=(t, 0,0,0)$, where $t$ is the time measured by a clock co-moving with the observer.

2. The metric expressed in proper non-inertial co-ordinates has the property

$$
g_{\mu \nu}(t, 0,0,0)=\eta_{\mu \nu}
$$

3. The connections $\Gamma_{\beta \gamma}^{\alpha}$ vanish at $(t, 0,0,0)$ if and only if the trajectory is a geodesic and there is no rotation.

The general geometrical construction of proper non-inertial co-ordinates is not very useful for practical calculations. However, in flat space-time, proper non-inertial co-ordinates can be constructed in an alternative way, more useful for practical calculations [2]. Here I present an elegant form of this construction introduced in [3].

Let $S$ be a Lorentz frame and let $S^{\prime}$ be the proper non-inertial frame of the observer whose 3 -velocity is $u^{i}\left(t^{\prime}\right) \equiv \mathbf{u}\left(t^{\prime}\right)$, as seen by an observer in $S$. The co-ordinate transformation between these two frames can be obtained by summing the infinitesimal Lorentz transformations. Let

$$
x^{\mu}=f^{\mu}\left(t^{\prime}, \mathbf{x}^{\prime} ; \mathbf{u}\right)
$$

denotes the ordinary Lorentz transformation, i.e. the transformation between two Lorentz frames specified by the constant relative velocity $\mathbf{u}$. The quantities

$$
f_{\nu}^{\mu}=\left(\frac{\partial f^{\mu}}{\partial x^{\prime \nu}}\right)_{\mathbf{u}=\text { const }}
$$

have explicit values

$$
\begin{gathered}
f_{0}^{0}=\gamma, \quad f_{j}^{0}=-\gamma u_{j}, \quad f_{0}^{i}=\gamma u^{i} \\
f_{j}^{i}=\delta_{j}^{i}+\frac{1-\gamma}{\mathbf{u}^{2}} u^{i} u_{j}
\end{gathered}
$$

where $u^{j}=-u_{j}, \mathbf{u}^{2}=u^{i} u^{i}, \gamma=1 / \sqrt{1-\mathbf{u}^{2}}$. The differential of (2) is

$$
d x^{\mu}=f_{\nu}^{\mu}\left(t^{\prime}, \mathbf{x}^{\prime} ; \mathbf{u}\right) d x^{\prime \nu}
$$


The transition to a noninertial frame introduces a time-dependent velocity: $\mathbf{u} \rightarrow \mathbf{u}\left(t^{\prime}\right)$. The transformation between $S$ and $S^{\prime}$ is given by the integration of (5) in the following way:

$$
x^{\mu}=\int_{0}^{t^{\prime}} f_{0}^{\mu}\left(t^{\prime}, 0 ; \mathbf{u}\left(t^{\prime}\right)\right) d t^{\prime}+\int_{C} f_{i}^{\mu}\left(t^{\prime}, \mathbf{x}^{\prime} ; \mathbf{u}\left(t^{\prime}\right)\right) d x^{i} .
$$

In (6), $C$ is an arbitrary curve with constant $t^{\prime}$, starting from 0 and ending at $x^{\prime i}$.

In general, $S^{\prime}$ can also rotate i.e. change the orientation of the co-ordinate axes with respect to an inertial frame. The rotation can be described by the rotation matrix $A_{j i}\left(t^{\prime}\right)=$ $-A_{j}^{i}\left(t^{\prime}\right)$. It satisfies the differential equation

$$
\frac{d A_{i j}}{d t}=-A_{i}^{k} \omega_{k j}
$$

where $\omega_{i k}=\varepsilon_{i k l} \omega^{l}, \varepsilon_{123}=1$ and $\omega^{i}\left(t^{\prime}\right)$ is the angular velocity as seen by an observer in $S$. In this more general case the transformation is also given by (6), but now $C$ is an arbitrary curve with constant $t^{\prime}$, starting from 0 and ending at $-A_{j}^{i}\left(t^{\prime}\right) x^{\prime j}$. Note that the proper noninertial co-ordinates $x^{\prime \mu}$ are constructed such that the space origins of $S$ and $S^{\prime}$ coincide at $t=t^{\prime}=0$.

The metric tensor in $S^{\prime}$ is given by

$$
g_{\mu \nu}^{\prime}=\frac{\partial x^{\alpha}}{\partial x^{\prime \mu}} \frac{\partial x^{\beta}}{\partial x^{\prime \nu}} g_{\alpha \beta}
$$

where $g_{\alpha \beta}=\eta_{\alpha \beta}=\operatorname{diag}(1,-1,-1,-1)$ is the Minkowski metric in $S$. Using (6), it is straightforward (but slightly tedious) to obtain

$$
\begin{gathered}
g_{i j}^{\prime}=-\delta_{i j}, \quad g_{0 j}^{\prime}=-\left(\boldsymbol{\omega}^{\prime} \times \mathbf{x}^{\prime}\right)_{j}, \\
g_{00}^{\prime}=c^{2}\left(1+\frac{\mathbf{a}^{\prime} \cdot \mathbf{x}^{\prime}}{c^{2}}\right)^{2}-\left(\boldsymbol{\omega}^{\prime} \times \mathbf{x}^{\prime}\right)^{2},
\end{gathered}
$$

where

$$
\omega^{\prime i}=\gamma\left(\omega^{i}-\Omega^{i}\right), \quad a^{i}=\gamma^{2}\left[a^{i}+\frac{1}{\mathbf{u}^{2}}(\gamma-1)(\mathbf{u} \cdot \mathbf{a}) u^{i}\right],
$$

$\Omega^{i}$ is the time-dependent Thomas precession frequency

$$
\Omega_{i}=\frac{1}{2 \mathbf{u}^{2}}(\gamma-1) \varepsilon_{i k j}\left(u^{k} a^{j}-u^{j} a^{k}\right),
$$

and $a^{i}=d u^{i} / d t$ is the time-dependent acceleration.

From Property 2 we see that the space co-ordinates $x^{i}$ are a generalization of Cartesian co-ordinates. However, this does not imply that an observer is not allowed to use polar co-ordinates, for example. The most general co-ordinate transformations that correspond merely to a redefinition of the co-ordinates of the same physical observer are the so-called restricted internal transformations [3], i.e. the transformations of the form

$$
t^{\prime}=f^{0}(t), \quad x^{i}=f^{i}\left(x^{1}, x^{2}, x^{3}\right),
$$


where $t, x^{i}$ are proper non-inertial co-ordinates. The quantities $g_{00} d t^{2}$ and

$$
d l^{2}=-g_{i j} d x^{i} d x^{j}
$$

do not change under restricted internal transformations. In order to describe physical effects as seen by a local observer, one must use proper non-inertial co-ordinates modulo restricted internal transformations. For simplicity, in the rest of the paper I use proper non-inertial co-ordinates.

Two observers with different trajectories have different proper non-inertial frames. In particular, this implies that even if there is no relative motion between two observers, they belong to different frames if they do not have the same position. This fact was not realized in many previous papers, which led to many misinterpretations. At first sight, this fact contradicts the well-known fact that two inertial observers in flat space-time may be regarded as belonging to the same Lorentz frame if there is no relative motion between them. However, this is because their proper non-inertial frames (with the space origins at their positions) are related by a translation of the space origin, which is a restricted internal transformation. In general, for practical purposes, two observers can be regarded as belonging to the same proper non-inertial frame if there is no relative motion between them and the other observer is close enough to the first one, in the sense that the metric expressed in proper non-inertial co-ordinates of the first observer does not depart significantly from $\eta_{\mu \nu}$ at the position of the second one. It is an exclusive property of Minkowski co-ordinates, among other proper noninertial co-ordinates in flat or curved space-time, that the metric is equal to $\eta_{\mu \nu}$ everywhere. This implies that two observers at different positions but with zero relative velocity may be regarded as belonging to the same co-ordinate frame only if they move inertially in flat space-time.

\section{Application to rotation}

For motivation, let us first review the problems [3] of the standard resolution [4, 5] of the Ehrenfest paradox. We study a rotating ring in a rigid non-rotating circular gutter with radius $r$. One introduces the co-ordinates of the rotating frame $S^{\prime}$

$$
\varphi^{\prime}=\varphi-\omega t, \quad r^{\prime}=r, \quad z^{\prime}=z, \quad t^{\prime}=t
$$

where $\varphi, r, z, t$ are cylindrical co-ordinates of the inertial frame $S$ and $\omega$ is the angular velocity. The metric in $S^{\prime}$ is given by

$$
d s^{2}=\left(c^{2}-\omega^{2} r^{\prime 2}\right) d t^{\prime 2}-2 \omega r^{\prime 2} d \varphi^{\prime} d t^{\prime}-d r^{\prime 2}-r^{\prime 2} d \varphi^{\prime 2}-d z^{\prime 2}
$$

It is generally accepted that the space line element should be calculated by the formula [6]

$$
d l^{\prime 2}=\gamma_{i j}^{\prime} d x^{i} d x^{\prime j}, \quad i, j=1,2,3
$$

where

$$
\gamma_{i j}^{\prime}=\frac{g_{0 i}^{\prime} g_{0 j}^{\prime}}{g_{00}^{\prime}}-g_{i j}^{\prime}
$$


This leads to the circumference of the ring

$$
L^{\prime}=\int_{0}^{2 \pi} \frac{r^{\prime} d \varphi^{\prime}}{\sqrt{1-\omega^{2} r^{\prime 2} / c^{2}}}=\frac{2 \pi r^{\prime}}{\sqrt{1-\omega^{2} r^{\prime 2} / c^{2}}} \equiv \gamma\left(r^{\prime}\right) 2 \pi r^{\prime}
$$

The circumference of the same ring as seen from $S$ is $L=2 \pi r=2 \pi r^{\prime}$. Since the ring is constrained to have the same radius $r$ as the same ring when it does not rotate, $L$ is not changed by the rotation, but the proper circumference $L^{\prime}$ is larger than the proper circumference of the non-rotating ring. This implies that there are tensile stresses in the rotating ring. The problem is that it is assumed here that (14) defines the proper frame of the whole ring. This implies that an observer on the ring sees that the circumference is $L^{\prime}=\gamma L$. The circumference of the gutter seen by him cannot be different from the circumference of the ring seen by him, so the observer on the ring sees that the circumference of the relatively moving gutter is larger than the proper circumference of the gutter, whereas we expect, using our experience with the usual Lorentz contraction, that he should see that it is smaller. Of course, it depends on how the circumference is measured. Here we have in mind an experimental procedure that in principle can also be used to measure the usual Lorentz contraction, based on photographing with a very short exposition, such that the change of the photographed object position during the exposition can be neglected. The size of the object's picture on the photography corresponds to the measured size. Obviously, with such a measuring procedure, for any observer the apparent circumference of the whole ring must be equal to the apparent circumference of the whole gutter. This is a simple consequence of the fact that, at any instance of time, any part of the ring is somewhere inside the gutter and any part of the gutter has a part of the ring near it. (Note also that this is not so for a well known "paradox" of a car in a garrage where different observers may disagree on whether a fast car can fit into an open garrage at rest. This is because, for each part of the car, there are times for which that part is outside the garrage as well as times for which it is inside the garrage.)

The problem discussed above resolves when one realizes that (14) does not define the proper frame of the ring. Each point on the ring belongs to a different proper non-inertial frame. The co-ordinates (14) are actually proper non-inertial co-ordinates (modulo a restricted internal transformation) of an observer that rotates in the centre of the ring. However, this raises another problem. If (16) is the correct definition of the space line element, then the observer that rotates in the centre should see that the circumference of the gutter is larger than the proper circumference of the gutter by a factor $\gamma\left(r^{\prime}\right)$. However, $\omega r^{\prime} / c$ can be arbitrarily large, so $\gamma\left(r^{\prime}\right)$ can be not only arbitrarily large, but also even imaginary. On the other hand, we know from everyday experience that the apparent velocity $\omega r^{\prime}$ of stars, due to our rotation, can exceed the velocity of light, but we see neither a contraction nor an elongation of the stars observed. This implies that the definition of the space line element (16) is not always appropriate. In [6], (16) is derived by defining the space line element through a measuring procedure that lasts a finite time, so, in general, this formula is not appropriate for a definition of the instantaneous length. Of course, if rotation is stationary, then it is not necessary to use a definition appropriate for the instantaneous length. However, we want to have a definition that is appropriate for any case and to consider a stationary rotation only as a special case of arbitrary motion. In flat space-time, as shown in [3], if physics is 
described by proper non-inertial co-ordinates modulo restricted internal transformations, a more appropriate definition of the space line element is (13).

Using the co-ordinate transformation (6), one can study the relativistic contraction in the same way as in the conventional approach with Lorentz frames. One assumes that two ends of a body are seen to have the same time co-ordinate. From (6) and (4) one can easily see that the co-ordinate transformation is linear in $x^{\prime i}$. As demonstrated in more detail in [3, 7], it implies that an arbitrarily accelerated and rotating observer sees equal lengths of other differently moving objects as an inertial observer whose instantaneous position and velocity are equal to that of the arbitrarily accelerated and rotating observer.

Using (6), one can also study the rate of clocks as seen by various observers. In particular, one can study the twin paradox for various motions of the observers [8]. However, it is more interesting to study not only the time shift after the two differently moving observers eventually meet, but also the continuous changes of the time shifts during the motion. As can be seen from (6), it is the time dependence (not the space dependence) of the co-ordinate transformation that significantly differs from the ordinary Lorentz transformations. One cannot invert (6) simply by putting $u^{i} \rightarrow-u^{i}$. Therefore, inertial and non-inertial observers see quite different continuous changes of the time shift.

Following [3], let us discuss in more detail the case of uniform circular motion. Here we only present the results, while the technical details are delegated to the Appendix. Assume that there are three clocks. One is at rest in $S$, so it moves inertially. The other two are moving around a circle with the radius $R$ and have the velocity $\omega R$ in the counter-clockwise direction, as seen by the observer in $S$. The relative angular distance between these two noninertial clocks is $\Delta \varphi_{0}$, as seen in $S$. The inertial observer will see that the two non-inertial clocks lapse equally fast, so I choose that he sees that they show the same time. He will see the clock rate $t=\gamma t^{\prime}$, where $\gamma=1 / \sqrt{1-\omega^{2} R^{2} / c^{2}}$. The observer co-moving with one of the non-inertial clocks will not see that the other non-inertial clock shows the same time as his clock. He will see the constant time shift given by the equation

$$
\gamma \omega\left(t^{\prime \prime}-t^{\prime}\right)=\beta^{2} \sin \left(\gamma \omega\left(t^{\prime \prime}-t^{\prime}\right)+\Delta \varphi_{0}\right)
$$

where $\beta^{2} \equiv \omega^{2} R^{2} / c^{2}$ and $t^{\prime \prime}$ is the time of the other non-inertial clock. Finally, let us see how the inertial clock looks like from the point of view of the observer co-moving with one of the non-inertial clocks. Let the position of the inertial clock be given by its co-ordinates $(x, y)$. We choose the origin of $S$ such that, at $t=t^{\prime}=0$, the space origins of $S$ and $S^{\prime}$ coincide and the velocity of the non-inertial observer is in the $y$-direction, as seen in $S$. The rate of clocks as seen by the non-inertial observer is given by

$$
t=\gamma t^{\prime}+\frac{\omega R}{c^{2}}\left[y \cos \gamma \omega t^{\prime}-(x+R) \sin \gamma \omega t^{\prime}\right] .
$$

The oscillatory functions in (20) vanish when they are averaged over time. This means that the observer in $S^{\prime}$ agrees with the observer in $S$ that the clock in $S^{\prime}$ is slower, but only in a time-averaged sense. For example, when these two clocks are very close to each other, then, by expanding (20) for small $t^{\prime}$, one finds $t=t^{\prime} / \gamma$, which is the result that one would obtain if the velocity of the non-inertial observer were constant. If the clock in $S$ is in the centre, which corresponds to $x=-R, y=0$, then (20) gives $t=\gamma t^{\prime}$, so in this case there is no oscillatory behaviour. 
Finally, let us shortly discuss the implications on the velocity of light. If (14) is interpreted as a proper frame of all observers on a rotating platform, then one can conclude that the observer on the rotating platform will see that the velocity of light depends on whether light is propagating in the clockwise or in the counter-clockwise direction (see, for example, [9]). This is related to the fact that the metric (15) is not time orthogonal. However, now we know that each observer belongs to a different proper non-inertial frame, and from Property 2 we see that in the vicinity of any observer the metric is equal to the Minkowski metric $\eta_{\mu \nu}$. This implies that for any local observer the velocity of light is isotropic and is equal to $c$, provided that it is measured by propagating a light beam in a small neighbourhood of the observer. In particular, this leads to a slightly different interpretation of the Sagnac effect [3], in which the velocity of light as seen by the observer on the rotating platform depends on the instantaneous relative position of the light beam with respect to the observer. The details are presented in the Appendix.

\section{Acknowledgments}

The author is grateful to G. Rizzi for his editorial critical remarks that significantly improved this paper. This work was supported by the Ministry of Science and Technology of the Republic of Croatia under the contract No. 0098002.

\section{Appendix}

In this Appendix, we derive Eqs. (19) and (20) and discuss how the velocity of light apppears to an observer on the rim of a rotating platform. In these derivations, we follow [3].

Let the gutter be placed at the $z=0$ plane. We put the space origin of $S$ at a fixed point on the gutter, such that the $y$-axis is tangential to the gutter and the $x$-axis is perpendicular to the gutter at $\mathbf{x}=0$. In the rest of the analysis the $z$-co-ordinate can be suppressed. A part of the ring in the gutter can be considered as a short rod initially placed at $\mathbf{x}=0$ and uniformly moving along the gutter in the counterclockwise direction. The gutter causes a torque that provides that the rod is always directed tangentially to the gutter. Therefore,

$\omega=u / R$, where $u=\sqrt{\mathbf{u}^{2}}$ is the time independent speed of rod. Now, $\gamma=1 / \sqrt{1-\omega^{2} R^{2} / c^{2}}$ is also time independent. Since a clock in $S^{\prime}$ is at $\mathbf{x}^{\prime}=0$, the clock rate between a clock in $S$ and a clock in $S^{\prime}$ is given by $t=\gamma t^{\prime}$, as seen by an observer in $S$. We assume that, initially, the axes $x^{\prime}, y^{\prime}$ are parallel to the axes $x, y$, respectively. Therefore the velocity

$$
\mathbf{u}\left(t^{\prime}\right)=\omega R\left(-\sin \gamma \omega t^{\prime}, \cos \gamma \omega t^{\prime}\right)
$$

is always in the $y^{\prime}$-direction and the solution of (7) is

$$
A_{i j}\left(t^{\prime}\right)=\left(\begin{array}{cc}
\cos \gamma \omega t^{\prime} & \sin \gamma \omega t^{\prime} \\
-\sin \gamma \omega t^{\prime} & \cos \gamma \omega t^{\prime}
\end{array}\right)
$$

The transformation (6) reduces to

$$
\left(\begin{array}{l}
x \\
y
\end{array}\right)=\left(\begin{array}{cc}
\cos \gamma \omega t^{\prime} & -\gamma \sin \gamma \omega t^{\prime} \\
\sin \gamma \omega t^{\prime} & \gamma \cos \gamma \omega t^{\prime}
\end{array}\right)\left(\begin{array}{c}
x^{\prime} \\
y^{\prime}
\end{array}\right)+R\left(\begin{array}{c}
\cos \gamma \omega t^{\prime}-1 \\
\sin \gamma \omega t^{\prime}
\end{array}\right)
$$




$$
t=\gamma t^{\prime}+\frac{\gamma}{c^{2}} \omega R y^{\prime}
$$

In particular, at $t^{\prime}=0$ these transformations become

$$
x=x^{\prime}, \quad y=\gamma y^{\prime}, \quad t=\frac{\gamma u}{c^{2}} y^{\prime},
$$

which coincide with the ordinary Lorentz boost at $t^{\prime}=0$ for the velocity in the $y$-direction.

Let us now study how other parts of the ring appear to the observer on the ring. Since the rotation is uniform the result cannot depend on $t^{\prime}$, so without losing on generality, we evaluate this at $t^{\prime}=0$. We introduce polar co-ordinates $r, \varphi$, defined by

$$
y=r \sin \varphi, \quad R+x=r \cos \varphi,
$$

which are new space co-ordinates for $S$, with the origin in the center of the circular gutter. The angle $\varphi$ is a good label of the position of any part of the ring even in $S^{\prime}$. (To visualize this, one can draw angular marks on the gutter. The number of marks separating two points on the gutter or on the ring is a measure of the "angular distance" in any frame.) Let $S^{\prime \prime}$ be the frame of another part of the ring. The position of that part of the ring is $x^{\prime \prime}=y^{\prime \prime}=0$. The relative position of the space origin of $S^{\prime \prime}$ with respect to that of $S^{\prime}$ is given by the constant relative angle $\Delta \varphi_{0}$, as seen by an observer in $S$. In analogy with (23)-(24), we find that $S^{\prime \prime}$ is determined by

$$
\begin{gathered}
\left(\begin{array}{l}
x \\
y
\end{array}\right)=\left(\begin{array}{cc}
\cos \left(\gamma \omega t^{\prime \prime}+\Delta \varphi_{0}\right) & -\gamma \sin \left(\gamma \omega t^{\prime \prime}+\Delta \varphi_{0}\right) \\
\sin \left(\gamma \omega t^{\prime \prime}+\Delta \varphi_{0}\right) & \gamma \cos \left(\gamma \omega t^{\prime \prime}+\Delta \varphi_{0}\right)
\end{array}\right)\left(\begin{array}{c}
x^{\prime \prime} \\
y^{\prime \prime}
\end{array}\right) \\
+R\left(\begin{array}{c}
\cos \left(\gamma \omega t^{\prime \prime}+\Delta \varphi_{0}\right)-1 \\
\sin \left(\gamma \omega t^{\prime \prime}+\Delta \varphi_{0}\right)
\end{array}\right), \\
t=\gamma t^{\prime \prime}+\frac{\gamma}{c^{2}} \omega R y^{\prime \prime} .
\end{gathered}
$$

Let the labels $A, B$ denote the co-ordinates of the part of the ring that lie at $S^{\prime}$ and $S^{\prime \prime}$, respectively. Since the observer sees both parts of the ring at the same instant, we have $t_{A}^{\prime}=t_{B}^{\prime}=0$. Since $x_{B}^{\prime \prime}=y_{B}^{\prime \prime}=0$, from (27) we find

$$
y_{B}=R \sin \left(\gamma \omega t_{B}^{\prime \prime}+\Delta \varphi_{0}\right)
$$

and from (28)

$$
t_{B}=\gamma t_{B}^{\prime \prime}
$$

From $t_{B}^{\prime}=0$ and (25) it follows $t_{B}=\omega R y_{B} / c^{2}$, which, because of (30), can be written as $\gamma t_{B}^{\prime \prime}=\omega R y_{B} / c^{2}$. This, together with (29), leads to the equation that determines $t_{B}^{\prime \prime}$ :

$$
\gamma \omega t_{B}^{\prime \prime}=\beta^{2} \sin \left(\gamma \omega t_{B}^{\prime \prime}+\Delta \varphi_{0}\right)
$$

where $\beta^{2} \equiv \omega^{2} R^{2} / c^{2}$. Eq. (31) together with the fact that the rotation is uniform implies (19).

To see how the inertial clock appears to the observer on the ring, the transformations (23) and (24) are sufficient. From (23) one expresses $y^{\prime}$ as a function of $x, y$ and $t^{\prime}$ and puts this in (24). The result is given by (20). 
Let us now discuss how the velocity of light appears to the observer on the ring. Let the light beam move along the gutter. The trajectory of the light beam expressed in $S$-coordinates is given by

$$
y=R \sin \omega_{L} t, \quad x=R\left(-1+\cos \omega_{L} t\right),
$$

where $\omega_{L}= \pm c / R$. The plus and minus signs refer to the counterclockwise and clockwise propagated beams, respectively. Using (23), (24), and (32), one can eliminate $x, y, t$ and express $x^{\prime}, y^{\prime}$ as functions of $t^{\prime}$. The speed of light as seen by the observer in $S^{\prime}$ is

$$
v_{L}^{\prime}=\sqrt{\left(\frac{d x^{\prime}}{d t^{\prime}}\right)^{2}+\left(\frac{d y^{\prime}}{d t^{\prime}}\right)^{2}} .
$$

Expanding (23) and (32) for small $t^{\prime}$ and $t$, respectively, one can easily find $y^{\prime}= \pm c t^{\prime}+\mathcal{O}\left(t^{\prime 2}\right)$,

$x^{\prime}=\mathcal{O}\left(t^{\prime 2}\right)$, which means that the observer sees the velocity of light equal to $c$ when the light is at the same position as the observer, just as expected.

\section{References}

[1] C. W. Misner, K. S. Thorne and J. A. Wheeler, Gravitation (W. H. Freeman and Company, New York 1995).

[2] R. A. Nelson, J. Math. Phys. 28, 2379 (1987); Erratum: R. A. Nelson, J. Math. Phys. 35, 6224 (1994).

[3] H. Nikolić, Phys. Rev. A 61, 032109 (2000).

[4] Ø. Grфn, Am. J. Phys. 43, 869 (1975).

[5] Ф. Grфn, Int. J. Theor. Phys. 16, 603 (1977).

[6] D. Landau and E. M. Lifschitz, The Classical Theory of Fields (Pergamon, New York 1971).

[7] H. Nikolić, Am. J. Phys. 67, 1007 (1999).

[8] H. Nikolić, Found. Phys. Lett. 13, 595 (2000).

[9] R. D. Klauber, Found. Phys. Lett. 11, 405 (1998). 